\title{
Isolation and identification of flavonoids components from Pteris vittata L.
}

\author{
Li-jing Lin ${ }^{1}$, Xiao-bing Huang ${ }^{1}$ and Zhen-cheng Lv²
}

\begin{abstract}
Background: Pteris vittata L. is rich in flavonoids which exhibit different bioactivities. In order to investigate the flavonoids components of $P$. vittata $L$., extracts of the plant were isolated by column chromatography on silica gel and Sephadex LH-20.

Results: Four flavonoids compounds were obtained, the structures were identified as kaempferol (1), quercetin (2), kaempferol-3-O-D-glucopyranoside (3) and rutin (4), respectively, on the basis of NMR spectroscopic analyses.

Conclusions: Compound $\mathbf{3}$ was reported for the first time from P. vittata $\mathrm{L}$.

Keywords: Pteris vittata L., Flavonoids, Kaempferol-3-O-D-glucopyranoside
\end{abstract}

\section{Background}

Pteris vittata L., a common fern known as 'Chinese Brake Fern', is native from China and widespread all over the world (Ma et al. 2001). It received much attentions in recent years because it was known to be a hyperaccumulator plant of arsenic used in phytoremediation (Cesaro et al. 2015; Tisarum et al. 2015; de Oliveira et al. 2016; Tiwari et al. 2016). It is also widely used in traditional Chinese medicine for diverse therapeutic applications, such as the treatment of influenza, dysentery, rheumatism, injury and scabies (Xie 1996). Previous qualitative phytochemical screening studies on $P$. vittata $\mathrm{L}$. have showed a substantial amount of flavonoids (Ding et al. 2009; Zhou et al. 2010). Compounds like leucocyanidin, leucodelphinidin, flavone ester apigenin 7-O-p-hydroxybenzoate, as well as a number of glycosides of apigenin, leutolin, isocutellarein-8-O-methyl-ether, kaempherol and quercetin were isolated from this plant in the past years (Salatino and Prado 1998; Imperato 2006). But, it attracts little attention on its chemical constituents or bioactivities recently. Numerous reports showed that flavonoids exhibited different bioactivities, such

\footnotetext{
*Correspondence: szsky@126.com

2 Department of Life Science, Huizhou University, Huizhou 516007, China Full list of author information is available at the end of the article
}

as anti-inflammatory, anti-oxidative, hypolipidemic, or antitumor effects (Bao et al. 2016; Feng et al. 2014; Matias et al. 2014; Raman et al. 2016). Plants are one of the important sources for screening active compounds. Hence, we attempt to obtain flavonoids components from $P$. vittata $\mathrm{L}$. to provide more information about its chemical constituents in this experiment.

\section{Methods}

\section{Plant material}

The whole plant of $P$. vittata $\mathrm{L}$. was collected from Guangzhou, China, in October 2015 and identified by Prof. P. T. Li (College of Forestry, South China Agricultural University, Guangzhou, P. R. China). The voucher specimen (No. PGZ090239) has been deposited at the CANT Herbarium, South China Agricultural University, Guangzhou, P. R. China.

\section{Experimental material}

Silica gel (100-200 and 200-300 mesh) for column chromatography (CC) and $\mathrm{GF}_{254}$ silica gel for thin layer chromatography (TLC) were purchased from Qingdao Marine Chemical Ltd., Qingdao, China. Sephadex LH-20 obtained from Amersham Biosciences, Sweden was used for CC. Other reagents were of analytical grade purchased from Guangzhou reagent Co. Ltd. NMR spectra 
$\left({ }^{1} \mathrm{H},{ }^{13} \mathrm{C}-\mathrm{NMR}\right)$ were determined on a Bruker AV-600 instrument using TMS as an internal reference.

\section{Extraction and isolation}

The whole plant $(7.0 \mathrm{~kg})$ of $P$. vittata $\mathrm{L}$. was extracted with methanol for three times at $25^{\circ} \mathrm{C}$. After the solvent was removed under vacuum, the concentrated extract was further extracted successively with petroleum ether, ethyl acetate and $n$-butanol. The ethyl acetate extract $(80 \mathrm{~g})$ was subjected to silica gel column chromatography using gradient hexane-EtOAc (50:1-1:1) to obtain 6 fractions. Fraction $2(12 \mathrm{~g})$ was subjected to silica gel column chromatography eluted with gradient hexane-acetone (100:1-10:1) to afford compound 1 (52 mg). Fraction 3 $(8 \mathrm{~g})$ was subjected repeatedly to silica gel column chromatography eluted with hexane-acetone (100:5) and was finally purified by column chromatography on Sephadex LH-20 eluted with $\mathrm{CHCl}_{3}-\mathrm{MeOH}$ (1:1) to give compound $2(53 \mathrm{mg})$. Fraction $5(2 \mathrm{~g})$ was subjected to silica gel column chromatography eluted with $\mathrm{CHCl}_{3}-\mathrm{MeOH}$ (95:5) to give compound 3 (36 mg). Fraction 6 (5 g) was subjected to silica gel column chromatography eluted with $\mathrm{CHCl}_{3}-\mathrm{MeOH}$ (10:1) led to the isolation of compound $4(280 \mathrm{mg})$.

\section{Results}

Four compounds were isolated and their structures were identified as kaempferol (1), quercetin (2), kaempferol-3-O-D-glucopyranoside (3) and rutin (4), respectively, by spectral analysis and comparison with the spectroscopic data reported in previous literatures (Fig. 1). NMR spectra data of the compounds were listed as follows (Additional file 1).

Compound 1 was obtained as yellow amorphous powder. ${ }^{1} \mathrm{H}-\mathrm{NMR}\left(600 \mathrm{MHz}, \mathrm{DMSO}-d_{6}\right) \delta_{\mathrm{H}}: 6.19(1 \mathrm{H}$, d, $J=1.8 \mathrm{~Hz}), 6.44(1 \mathrm{H}, \mathrm{d}, J=1.8 \mathrm{~Hz}), 6.92(2 \mathrm{H}, \mathrm{d}$, $J=9.0 \mathrm{~Hz}), 8.04(2 \mathrm{H}, \mathrm{d}, J=9.0 \mathrm{~Hz}) \cdot{ }^{13} \mathrm{C}-\mathrm{NMR}(150 \mathrm{MHz}$, DMSO- $\left.d_{6}\right) \delta_{\mathrm{C}}$ : $93.5(\mathrm{C}-8), 98.2(\mathrm{C}-6), 103.0(\mathrm{C}-10), 115.4$ (C-3', C-5'), $121.7\left(\mathrm{C}-1^{\prime}\right), 129.5\left(\mathrm{C}-2^{\prime}\right), 130.5\left(\mathrm{C}-6^{\prime}\right), 135.6$ (C-3), 146.8 (C-2), 156.2 (C-5), 159.2 (C-4'), 160.7 (C-9), $163.9(\mathrm{C}-7), 175.9$ (C-4). It was determined as kaempferol by comparison with the spectroscopic data reported in the literature (Liu et al. 2009).

Compound 2 was obtained as yellow amorphous powder. ${ }^{1} \mathrm{H}-\mathrm{NMR} \quad\left(600 \mathrm{MHz}, \quad \mathrm{DMSO}-d_{6}\right) \quad \delta_{\mathrm{H}}: 6.19$ $(1 \mathrm{H}, \mathrm{d}, J=1.8 \mathrm{~Hz}), 6.40(1 \mathrm{H}, \mathrm{d}, J=2.4 \mathrm{~Hz}), 6.88(1 \mathrm{H}$, $\mathrm{d}, J=8.4 \mathrm{~Hz}), 7.54(1 \mathrm{H}, \mathrm{dd}, J=2.4,8.4 \mathrm{~Hz}), 7.67(1 \mathrm{H}$, d, $J=2.4 \mathrm{~Hz}) .{ }^{13} \mathrm{C}-\mathrm{NMR}\left(150 \mathrm{MHz}\right.$, DMSO- $\left.d_{6}\right) \delta_{\mathrm{C}}: 93.3$ (C-8), 98.1 (C-6), 102.9 (C-10), 114.9 (C-2'), 115.5 (C-5'),<smiles>O=c1c(O)c(-c2ccc(O)cc2)oc2cc(O)cc(O)c12</smiles>

kaempferol (1)<smiles>O=c1c(OC2CCCCC2)c(-c2ccc(O)cc2)oc2cc(O)cc(O)c12</smiles>

kaempferol-3-O-D-glucopyranoside (3)<smiles>O=c1c(O)c(-c2ccc(O)c(O)c2)oc2cc(O)cc(O)c12</smiles>

quercetin (2)

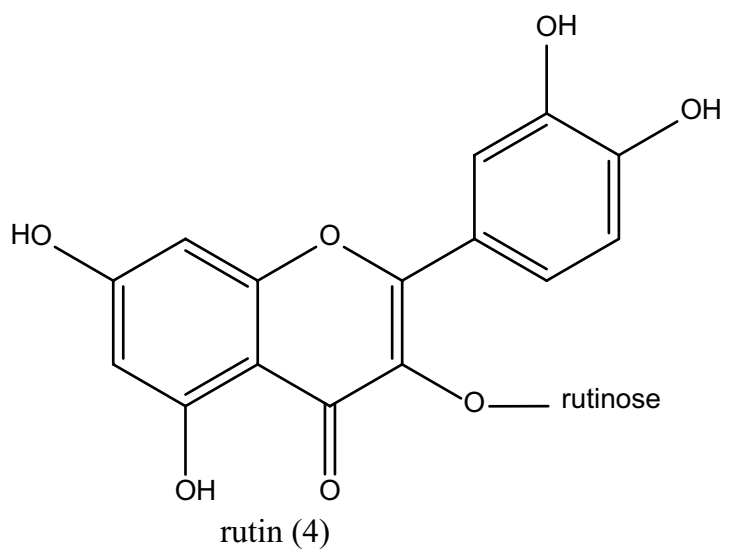

Fig. 1 Structures of flavonoids from P. vittata $L$ 
$119.9\left(\mathrm{C}-6^{\prime}\right), 121.8\left(\mathrm{C}-1^{\prime}\right), 135.6(\mathrm{C}-3), 144.9\left(\mathrm{C}-1^{\prime}\right), 146.7$ (C-1), 147.6 (C-4'), 156.1 (C-5), 160.7 (C-9), 163.8 (C-7), 175.8 (C-4). It was identified as quercetin by comparison with the spectroscopic data reported in the literature $\mathrm{Ma}$ et al. 2007).

Compound 3 was obtained as yellow amorphous powder. ${ }^{1} \mathrm{H}-\mathrm{NMR}\left(600 \mathrm{MHz}, \mathrm{DMSO}-d_{6}\right) \delta_{\mathrm{H}}: 3.09(2 \mathrm{H}$, d, $J=4.2 \mathrm{~Hz}), 3.21(1 \mathrm{H}, \mathrm{d}, J=7.8 \mathrm{~Hz}), 3.32(1 \mathrm{H}, \mathrm{d}$, $J=11.4 \mathrm{~Hz}), 3.55(1 \mathrm{H}, \mathrm{d}, J=11.4 \mathrm{~Hz}), 4.29(1 \mathrm{H}, \mathrm{s}), 4.96$ $(1 \mathrm{H}, \mathrm{s}), 5.07(1 \mathrm{H}, \mathrm{s}), 5.36(1 \mathrm{H}, \mathrm{s}), 5.46(1 \mathrm{H}, \mathrm{d}, J=7.8), 6.21$ $(1 \mathrm{H}, \mathrm{d}, J=1.8 \mathrm{~Hz}), 6.43(1 \mathrm{H}, \mathrm{d}, J=1.8 \mathrm{~Hz}), 6.88(2 \mathrm{H}, \mathrm{d}$, $J=9.0 \mathrm{~Hz}), 8.04(2 \mathrm{H}, \mathrm{d}, J=9.0 \mathrm{~Hz}) .{ }^{13} \mathrm{C}-\mathrm{NMR}(150 \mathrm{MHz}$, DMSO- $\left.d_{6}\right) \delta_{\mathrm{C}}$ : $61.1\left(\mathrm{C}-6^{\prime \prime}\right), 70.2\left(\mathrm{C}-4^{\prime \prime}\right), 74.5\left(\mathrm{C}-2^{\prime \prime}\right), 76.8$ $\left(\mathrm{C}-5^{\prime \prime}\right), 77.8\left(\mathrm{C}-3^{\prime \prime}\right), 94.0$ (C-8), $99.1(\mathrm{C}-6), 101.2\left(\mathrm{C}-1^{\prime \prime}\right)$, $104.3(\mathrm{C}-10), 115.4\left(\mathrm{C}-3^{\prime}, \mathrm{C}-5^{\prime}\right), 121.2\left(\mathrm{C}-1^{\prime}\right), 131.2\left(\mathrm{C}-2^{\prime}\right.$, C-6'), 133.5 (C-3), 156.6 (C-2), 156.7 (C-9), 160.3 (C-4'), 161.5 (C-5), 164.5 (C-7), 177.8 (C-4). It was determined as kaempferol-3-O-D-glucopyranoside by comparison with the spectroscopic data reported in the literature (Long et al. 2011).

Compounds 4 was obtained as yellow amorphous powder. ${ }^{1} \mathrm{H}-\mathrm{NMR}\left(600 \mathrm{MHz}, \mathrm{DMSO}-d_{6}\right) \delta_{\mathrm{H}}: 1.00(3 \mathrm{H}, \mathrm{d}$, $J=6.0 \mathrm{~Hz}), 3.08(3 \mathrm{H}, \mathrm{m}), 3.70(1 \mathrm{H}, \mathrm{d}, J=12.0 \mathrm{~Hz}), 4.39$ $(1 \mathrm{H}, \mathrm{d}, J=2.4 \mathrm{~Hz}), 5.10(2 \mathrm{H}, \mathrm{d}, J=18.0 \mathrm{~Hz}), 5.28(1 \mathrm{H}$, s), $5.35(1 \mathrm{H}, \mathrm{d}, J=6.0 \mathrm{~Hz}), 6.20(1 \mathrm{H}, \mathrm{d}, J=2.4 \mathrm{~Hz}), 6.39$ $(1 \mathrm{H}, \mathrm{d}, J=2.4 \mathrm{~Hz}), 7.54(1 \mathrm{H}, \mathrm{d}, J=2.4 \mathrm{~Hz}), 7.56(1 \mathrm{H}$, dd, $J=2.4,12.0 \mathrm{~Hz}){ }^{13} \mathrm{C}-\mathrm{NMR}\left(150 \mathrm{MHz}\right.$, DMSO- $\left.d_{6}\right)$ : $\delta_{\mathrm{C}}: 17.6\left(\mathrm{C}-6^{\prime \prime \prime}\right), 66.9\left(\mathrm{C}-6^{\prime \prime}\right), 68.1\left(\mathrm{C}-5^{\prime \prime \prime}\right), 69.9\left(\mathrm{C}-4^{\prime \prime}\right)$, $70.2\left(\mathrm{C}-2^{\prime \prime \prime}\right), 70.4\left(\mathrm{C}-3^{\prime \prime \prime}\right), 71.7\left(\mathrm{C}-4^{\prime \prime \prime}\right), 73.9\left(\mathrm{C}-2^{\prime \prime}\right), 75.8$ (C-5"), 76.3 (C-3"), 93.5 (C-8), 98.5 (C-6), $100.6\left(\mathrm{C}-1^{\prime \prime \prime}\right)$, $101.0\left(\mathrm{C}-1^{\prime \prime}\right), 103.8$ (C-10), 115.1 (C-2'), $116.1\left(\mathrm{C}-5^{\prime}\right)$, $121.0\left(\mathrm{C}-6^{\prime}\right), 121.5\left(\mathrm{C}-1^{\prime}\right), 133.2(\mathrm{C}-3), 144.6\left(\mathrm{C}-3^{\prime}\right), 148.3$ (C-4'), 156.3 (C-9), 156.5 (C-2), 161.1 (C-5), 164.0 (C-7), 177.2 (C-4). It was identified as rutin compared with the spectroscopic data reported in the literature (Zhang et al. 2010).

\section{Conclusion}

Phytochemical screening of $P$. vittata $\mathrm{L}$. was carried out. Four flavonoids were obtained and identified as kaempferol (1), quercetin (2), kaempferol-3-O-D-glucopyranoside (3) and rutin (4), respectively. Compound 1, 2 and 4 were known components which were mentioned in the previous results (Imperato and Telesca 2000; Imperato 2006). Compound 3 was a derivative of kaempferol with a glucopyranoside. It is reported for the first time from $P$. vittata $\mathrm{L}$. The current results may provide more information about flavonoids profiles of this plant.

\section{Additional file}

Additional file 1. NMR spectra data of the compounds can be found online as Additional file for the present article.

\section{Authors' contributions}

LZC designed the experiment and prepared the manuscript. HXB isolated and purified the flavonoid components. LL identified structures of the compounds. All authors read and approved the final manuscript.

\section{Author details}

${ }^{1}$ Agricultural Product Processing Research Institute, Chinese Academy of Tropical Agricultural Sciences, Zhanjiang 524001, China. ${ }^{2}$ Department of Life Science, Huizhou University, Huizhou 516007, China.

\section{Acknowledgements}

This work was financially supported by Foundation for Distinguished Young Talents in Higher Education of Guangdong (2014KQNCX214) and Technology Research and Development Program of Huizhou (2014B040008001)

\section{Competing interests}

The authors declare that they have no competing interests.

Received: 24 April 2016 Accepted: 12 September 2016

Published online: 23 September 2016

\section{References}

Bao L, Hu L, Zhang Y, Wang YI (2016) Hypolipidemic effects of flavonoids extracted from Pteris vittata. Exp Ther Med 11(4):1417-1424

Cesaro P, Cattaneo C, Bona E, Berta G, Cavaletto M (2015) The arsenic hyperaccumulating Pteris vittata expresses two arsenate reductases. Sci Rep 5:14525

de Oliveira LM, Gress J, De J, Rathinasabapathi B, Marchi G, Chen Y, Ma LQ (2016) Sulfate and chromate increased each other's uptake and translocation in As-hyperaccumulator Pteris vittata. Chemosphere 147:36-43

Ding LJ, Su GL (2009) Extraction of flavonoids from ladder brake with microwave and its antioxidative activity. J Food Sci Bio. 28(5):623-626

Feng Z, Hao W, Lin X, Fan D, Zhou J (2014) Antitumor activity of total flavonoids from Tetrastigma hemsleyanum Diels et Gilg is associated with the inhibition of regulatory T cells in mice. Onco Targets Ther 7:947-956

Imperato F (2006) The new flavones ester Apigenin-7-O-oxy-p-hydroxybenzoate and 3-Di-C-glycosyl flavones from Pteris vittata. Am Fern J. 96(2):62-65

Imperato F, Telesca A (2000) 6-C-cellobiosylisoscutellarein-8-methyl ether, a new flavonoid from Pteris vittata. Am Fern J. 96(1):42-47

Liu CD, Chen J, Wang JH (2009) Confirmation of the structure of tiliroside, an acylated kaempferol glycoside, by ${ }^{13} \mathrm{C}$-Nuclear Magnetic Resonance. Chem Nat Comp. 45(6):808-810

Long F, Deng L, Chen Y (2011) Study on the chemical constituents in the flowers of Ligustrum lucium. West China J Pharm Sci. 26(2):97-100

Ma LQ, Komar KMN, Tu C, Zhang W, Cai Y, Kennelley E (2001) A fern that hyperaccumulates arsenic. Nature 409:579

Ma XM, Liu Y, Shi YP (2007) Phenolic derivatives with free-radical-scavenging activities from Ixeridium gracile (DC.) Shih. Chem Biodivers 4(9):2172-2181

Matias A, Nunes SL, Poejo J, Mecha E, Serra AT, Madeira PJ, Bronze MR, Duarte CM (2014) Antioxidant and anti-inflammatory activity of a flavonoid-rich concentrate recovered from Opuntia ficus-indica juice. Food Funct 5(12):3269-3280

Raman ST, Ganeshan AK, Chen C, Jin C, Li SH, Chen HJ, Gui Z (2016) In vitro and in vivo antioxidant activity of flavonoid extracted from mulberry fruit (Morus alba L.). Pharmacogn Mag 12(46):128-133

Salatino MLF, Prado J (1998) Flavonoid glycosides of Pteridaceae from Brazil. Biochem Syst Ecol. 26:761-769

Tisarum R, Chen Y, Dong X, LessI JT, Ma LQ (2015) Uptake of antimonite and antimonate by arsenic hyperaccumulator Pteris vittata: effects of chemical analogs and transporter inhibitor. Environ Pollut 206:49-55

Tiwari S, Sarangi BK, Thul ST (2016) Identification of arsenic resistant endophytic bacteria from Pteris vittata roots and characterization for arsenic remediation application. J Environ Manage 180:359-365

Xie WZ (1996) Compilation of national Chinese herbal medicine. People Health Press, Beijing, pp 646-647

Zhang S, Tao ZM, Zhang Y (2010) Chemical constituents from the stems and leaves of Elaeocarpus glabripetalus. Chin J Nat Med. 8(1):21-24

Zhou XJ, Yang ZQ, Jing HY, Gao YX (2010) Study on optimization of total flavonoids extraction technology in Pteris Vittata L. Res Dev Mark. 26(3):210-211 